\title{
Effects of Rifapentine and Rifampicin in Smear-positive Pulmonary Tuberculosis Patients and the Incidence Comparison of Complications
}

\author{
Jianmei $\mathrm{Ma}^{1}$, Hongyang $\mathrm{Shi}^{2}$ and Yan Zhang ${ }^{3}$ \\ ${ }^{1}$ Department of Diagnostics, College of Clinical Medical, Xi'an Medical University; Xi'an 710021 \\ China; \\ 2 Department of Respiration, the Second Hospital of Xi 'an Jiaotong University; Xi'an 710004 China; \\ ${ }^{3}$ Department of Pharmacognosy, College of Pharmacy, Xi'an Medical University; Xi'an 710021 \\ China.
}

Keywords: Smear-positive tuberculosis; rifapentine; rifampin; clinical effect; complications.

\begin{abstract}
Objective: to analyze the clinical effects and complications of rifapentine and rifampicin in patients with smear-positive pulmonary tuberculosis. Methods: from August 2013 to June 2017, 100 of the smear-positive pulmonary tuberculosis patients treated in our hospital were selected and divided into two groups according to treatment methods. Patients in the control group were treated with rifampicin + isoniazid + pyrazinamide + ethambutol, while patients in the observation group were treated with rifapentine + isoniazid + pyrazinamide + ethambutol. In addition to the nisus absorption rate, the cavity closure rate and the rate of sputum negative conversion after the treatment, the comparison was also applied on the level of peripheral blood T-lymphocyte subsets and the incidence of complications before and after the treatment. Results: compared with the control group, patients in the observation group had higher levels of CD8+, CD4+ and CD3+ in peripheral blood T lymphocyte subsets; the nisus absorption rate, cavity closure rate and the rate of sputum negative conversion in the observation group were higher after treatment, and the difference between the groups was statistically significant $(\mathrm{P}<0.05)$; the incidence of complications in the observation group was $6 \%$, which was lower than that in the control group $(22 \%)$. There was a statistically significant difference between the two groups $(\mathrm{P}<0.05)$. Conclusion: the clinical efficacy of rifapentine in smear-positive pulmonary tuberculosis patients is superior to that of rifampicin and therefore it worth being promoted.
\end{abstract}

\section{Introduction}

Tuberculosis is a common clinical chronic infectious disease and its main clinical symptoms include macritus, low fever, night sweats, fatigue, thoracalgia, choking sensation in chest, expectoration, cough, etc. [1-2]. After the onset of the disease, it can affect the lungs and other organs, and the incidence has increased significantly in recent years. Many people in China have infected with Mycobacterium tuberculosis, and about 5 million of them eventually evolved into tuberculosis, of which smear-positive tuberculosis patients account for 30\% [3-4]. At the present stage, the patients are usually treated with regular drug combination and the drugs commonly used include rifapentine, rifampin, isoniazid, pyrazinamide, ethambutol, etc. [5-6]. In this study, the rifapentine-based and rifampicin-based drug combinations were respectively used in the treatment of the two groups, and the application effects were compared and reported as follows.

\section{Data and Methods}

\subsection{Clinical Data.}

100 of the smear-positive tuberculosis patients who were treated in our hospital from August 2013 to June 2017 were selected as the objects of the study and all of them signed the informed consent form voluntarily. The inclusion criteria: 1) patients conformed to the diagnostic criteria for pulmonary tuberculosis; 2) the results of the tuberculin test were positive; 3) patients did not have any disturbance of consciousness and mental disorders; 4) patients did not have contraindications for the 
drugs used in this study; 5) patients could completed the whole treatment in the study. Exclusion criteria: 1) The patients were reluctant to participate; 2) the patients had disturbances of consciousness and mental disorders; 3 ) the patients were allergic to the drugs used in this study; 4) the patients were accompanied by severe organic diseases such as liver and kidney; 5) the patients were likely to drop out. The 100 patients were divided into two groups according to treatment methods. The patients in the observation group were aged between 22 and 65 and the average age was (39.68 \pm 6.38$)$ years old; their disease course was between 1 month and 18 months and the mean disease course was $(4.98 \pm 2.68)$ months; there were 50 cases in this group, including 39 males and 11 females. The patients in the control group were aged between 23 and 66 and the average age was (40.02 \pm 6.15$)$ years old; their disease course was between 1 month and 19 months and the mean disease course was $(5.02 \pm 2.18)$ months; there were 50 cases in this group, including 35 males and 15 females. This study was approved by the Hospital Ethics Committee. It can be seen from the comparison that the differences between the two sets of data is of comparative value, $\mathrm{P}>0.05$.

\subsection{Methods.}

The control group used the therapy of rifampin + isoniazid + pyrazinamide + ethambutol. Every morning, patients orally took 0.45g rifampicin (Approved No.: GUO YAO ZHUN ZI H41021799; Manufacturer: Kaifeng Pharmaceutical (Group) Co., Ltd.), 0.3g isoniazid (Approval No.: GUO YAO ZHUN ZI H21022350; Manufacturer: Shenyang Hongqi Pharmaceutical Co., Ltd.), 1.25g pyrazinamide (Approval No.: GUO YAO ZHUN ZI H51020877; Manufacturer: Chengdu Jinhua Pharmaceutical Co., Ltd.) and 0.75g ethambutol (Approval No.: GUO YAO ZHUN ZI H13020619; Manufacturer: Huabei Pharmaceutical Co., Ltd.), once a day for 3 months. 3 months later, the patients received consolidation therapy. The therapeutic regimen was rationally adjusted according to the patients' actual conditions. The dose of ethambutol and pyrazinamide was reduced and treatment was continued for 6 months.

The therapy adopted in the observation group was rifapentine + isoniazid + pyrazinamide + ethambutol. Every morning, patients orally took $0.6 \mathrm{~g}$ rifapentine (Approved No.: GUO YAO ZHUN ZI H10940012; Manufacturer: Shanxi Guorun Pharmaceutical Co., Ltd.). The way of taking isoniazid, pyrazinamide and ethambutol was same to that in the control group and patients received 3-month treatment. 3 months later, the patients received consolidation therapy. The therapeutic regimen was rationally adjusted according to the patients' actual conditions. The dose of ethambutol and pyrazinamide was reduced and treatment was continued for 6 months.

\subsection{Observation Targets.}

The levels of peripheral blood T-lymphocyte subsets before and after treatment were compared between the two groups of patients, including the $\mathrm{CD} 8^{+}, \mathrm{CD}^{+}$and $\mathrm{CD} 3^{+}$levels. $5 \mathrm{ml}$ peripheral blood of every patient in the two groups was collected both before and after the treatment, and the levels of each index were measured with flow cytometry and three-color analysis technique [7-8].

The nidus absorption rate, cavity closure rate and sputum negative conversion rate of the two groups after treatment were compared.

The complications of the two groups were compared, including gastrointestinal reactions, leukopenia, bush yaws and abnormal liver function.

\subsection{Statistical Analysis.}

SPSS20.0 statistical software was used to analyze the data and enumeration data was tested by the chi-square test, presented as ratio. Measurement data was analyzed by t-test and expressed as $(\bar{X} \pm \mathrm{s})$ and the difference was significant when $\mathrm{P}<0.05$.

\section{Results}

\subsection{Comparison of Peripheral Blood T Lymphocyte Subsets before and after Treatment in Patients of the Two Groups.}

Before treatment, there was no statistical significance between the two groups in the levels of $\mathrm{CD}^{+}, \mathrm{CD}^{+}$, and $\mathrm{CD}^{+}$in peripheral blood $\mathrm{T}$ lymphocyte subsets, $\mathrm{P}>0.05$; compared with the control group, patients in the observation group had higher levels of $\mathrm{CD}^{+}, \mathrm{CD}^{+}$and $\mathrm{CD} 3^{+}$in the peripheral 
blood $\mathrm{T}$ lymphocyte subsets after treatment. There were statistically significant differences between the two groups $(\mathrm{P}<0.05)$, as shown in Table 1:

Table. 1 Comparison of peripheral blood T-lymphocyte subsets before and after treatment in the two

\begin{tabular}{ccccccc}
\hline \multicolumn{1}{c}{ CD8+ } & \multicolumn{2}{c}{ groups $\left(X_{ \pm \mathrm{s}}, \%\right)$} \\
Group & $\begin{array}{c}\text { Before } \\
\text { treatment }\end{array}$ & $\begin{array}{c}\text { After } \\
\text { treatment }\end{array}$ & $\begin{array}{c}\text { Before } \\
\text { treatment }\end{array}$ & $\begin{array}{c}\text { After } \\
\text { treatment }\end{array}$ & $\begin{array}{c}\text { Before } \\
\text { treatment }\end{array}$ & $\begin{array}{c}\text { After } \\
\text { treatment }\end{array}$ \\
\hline $\begin{array}{c}\text { Observation } \\
\text { group (n=50) } \\
\text { Control }\end{array}$ & $24.56 \pm 2.65$ & $28.02 \pm 2.52$ & $22.95 \pm 2.85$ & $45.25 \pm 4.56$ & $43.69 \pm 3.59$ & $71.56 \pm 4.58$ \\
group (n=50) & $24.89 \pm 2.02$ & $25.02 \pm 2.05$ & $22.89 \pm 2.92$ & $32.52 \pm 2.02$ & $43.72 \pm 3.06$ & $52.63 \pm 4.05$ \\
$\mathrm{t}$ & 0.7003 & 6.5301 & 0.1039 & 18.0485 & 0.0449 & 21.8939 \\
$\mathrm{P}$ & 0.4854 & 0.0000 & 0.9174 & 0.0000 & 0.9642 & 0.0000 \\
\hline
\end{tabular}

3.2 The Comparison of Indus Absorption Rate, Cavity Closure Rate and Sputum Negative Conversion Rate between the Two Groups after the Treatment.

Compared with the control group, patients in the observation group presented better effects in the nidus absorption rate, cavity closure rate and the rate of sputum negative conversion after treatment, and the difference between the groups was statistically significant $(\mathrm{P}<0.05)$, as shown in Table 2 . Table. 2 The comparison of nidus absorption rate, cavity closure rate and sputum negative conversion rate between the two groups after the treatment (n, \%)

\begin{tabular}{ccccc}
\hline Group & $\begin{array}{c}\text { Number of } \\
\text { cases }(\mathrm{n})\end{array}$ & $\begin{array}{c}\text { Nidus absorption } \\
\text { rate }\end{array}$ & $\begin{array}{c}\text { Cavity closure } \\
\text { rate }\end{array}$ & $\begin{array}{c}\text { Sputum negative } \\
\text { conversion rate }\end{array}$ \\
\hline $\begin{array}{c}\text { Observation } \\
\text { group }\end{array}$ & 50 & $45(90.00)$ & $35(70.00)$ & $47(94.00)$ \\
$\begin{array}{c}\text { Control group } \\
\text { X2 }\end{array}$ & 50 & $36(72.00)$ & $25(50.00)$ & $37(74.00)$ \\
P & & 5.2632 & 4.1667 & 7.4405 \\
\end{tabular}

\subsection{Comparison of Complications between the Two Groups.}

The incidence of complications in the observation group was $6 \%$, lower than that in the control group $(22 \%)$, and there was a statistically significant difference between the two groups $(\mathrm{P}<0.05)$, as shown in Table 3.

Table. 3 Comparison of complications between the two groups (n, \%)

\begin{tabular}{ccccccc}
\hline Group & $\begin{array}{c}\text { Number of } \\
\text { cases (n) }\end{array}$ & $\begin{array}{c}\text { Gastrointestinal } \\
\text { reaction }\end{array}$ & Leukopenia & $\begin{array}{c}\text { Bush } \\
\text { yaws }\end{array}$ & $\begin{array}{c}\text { Abnormal } \\
\text { liver } \\
\text { function }\end{array}$ & Incidence \\
\hline $\begin{array}{c}\text { Control group } \\
\text { Observation }\end{array}$ & 50 & $1(2.00)$ & $3(6.00)$ & $\begin{array}{c}3 \\
(6.00)\end{array}$ & $4(8.00)$ & $11(22.00)$ \\
group & 50 & $1(2.00)$ & $0(0.00)$ & $\begin{array}{c}1 \\
(2.00)\end{array}$ & $1(2.00)$ & $3(6.00)$ \\
X2 & & & & & 5.3156 \\
P & & & & & & 0.0211 \\
\hline
\end{tabular}

\section{Discussion}

Tuberculosis is a common clinical infectious disease. It is a chronic granulation abscess caused by the infection of human lungs with Mycobacterium tuberculosis. If no effective measure is taken to prevent and control it in time, it may induce other various diseases and pose a serious threat to the patient's life and safety [9-10]. At the present stage, there are many ways to treat the disease clinically, among which drug therapy is used relatively more frequently and is beneficial to reduce the clinical infection rate and mortality of patients [11]. Rifampicin and rifapentine have been widely used in the 
treatment of smear-positive tuberculosis in recent years. By comparing the clinical efficacy of the two drugs, this study received results that, compared with the control group, patients of the observation group enjoyed good effects on the levels of $\mathrm{CD}^{+}, \mathrm{CD}^{+}$and $\mathrm{CD}^{+}$in peripheral blood $\mathrm{T}$ lymphocyte subsets as well as nidus absorption rate, cavity closure rate and sputum negative conversion rate; the incidence of complications in the observation group was $6 \%$, which was lower than that in the control group (22\%). This indicates that, compared with rifampin, rifapentine is more effective in treating smear-positive tuberculosis. Rifampicin is mainly derived from rifamycin and it can specifically bind to the RNA polymerase $\beta$ subunit in bacteria, thereby inhibiting the activity of bacteria and killing the bacteria. However, the use of this drug in treatment has a high incidence of complications, and patients with long-term use of this drug are likely to produce resistance, and ultimately it is difficult to obtain the desired therapeutic effect. Rifapentine is also a commonly used drug for the treatment of pulmonary tuberculosis in clinical practice. It is a cyclopentyl derivative which can kill Mycobacterium tuberculosis in various growth states and growth environments, with high drug safety. In addition, this drug has good antibacterial spectrum properties and can exert a good antibacterial effect against viruses, chlamydia, Mycobacterium tuberculosis, Leprosy and other microorganisms, and can be used to effectively treat smear-positive tuberculosis.

In summary, the clinical efficacy of rifapentine in patients with smear-positive tuberculosis is better than that of rifampicin, and thus it is worth being promoted.

\section{Acknowledgments}

Social Development Science and Technology Project of Shaanxi Provincial Science and Technology Department: "Effects of Cigarette Smoke on Serum CD8+T Cell in Patients with Chronic Obstructive Pulmonary Disease" (No. 2016SF-066).

\section{References}

[1]. Yang Jun, Wei Min. Comparison of Efficacy and Drug-induced Liver Injury in Patients with Pulmonary Tuberculosis Complicated with Chronic Liver Disease Treated with Rifampicin or Rifapentine Chemotherapy [J]. Chinese Hepatology, 2017, 22(1):55- 58.

[2]. He Tao. Clinical effect of chemotherapy regimens included levofloxacin and rifapentine on smear-positive pulmonary tuberculosis [J]. International Journal of Respiration, 2016, 36(24): $1855-1857$.

[3]. Yan Qionghong, $\mathrm{Wu}$ Xian. The treatment effects of rifapentine on patients with pulmonary tuberculosis and its effects on serum IL-6, IFN- $\gamma$ and CA125 [J]. Shaanxi Medical Journal, 2017, 46(12):1767-1768.

[4]. Lou Hay, Sun Qin. Rifapentin Daily Therapy for Tuberculosis: a Randomized, Dose-ranging Trial in Clinical Trials [J]. Chinese Journal of Antituberculosis, 2016, 38(11): 907-907.

[5]. Hong Xinhua, Chen Kai. Effects of Tuberculosis Drugs Combined with Rifapentine on the Immune Function, Inflammatory Factors, VEGF and MMP-9 of TB Patients [J]. Journal of Kunming Medical University, 2016, 37(2):111-114.

[6]. Zhang Jingo, Wang Lipping, Wan Yueqiang, et al. Neixiao Luoli pills combined magnesium isoglycyrrhizinate and Rifapentine treatment of pulmonary tuberculosis [J]. Journal of Changchun University of Traditional Chinese Medicine, 2016, 32(4):778-780.

[7]. Zhang Lanolin, Yang Hua, Xiao Heping, et al. Determination of in vitro synergy by a checkerboard method when 3 core antimicrobial agents of the retreatment new scheme combined against MDR-MTB and XDR-MTB [J]. Chinese Journal of Tuberculosis and Respiratory Diseases, 2016, 39(6):464-468. 
[8]. Zhu Yu. Evaluation on effect of soniazid combined with rifampicin treating patients with mono resistance to soniazid or rifampicin tuberculosis [J]. Journal of Clinical and Experimental Medicine, 2016, 13(13): 1297- 1299.

[9]. Due Xing, Qi Xing, Li Yangjun, et al. Study on the Effect of Procalcitonin, IL-15 and INF- $\gamma$ Levels of Serum in Patients with Pulmonary Tuberculosis of Levofloxacin and Rifampicin [J]. Progress in Modern Biomedicine, 2016, 16 (18):3523-3526.

[10]. Han Dan, Duane Hainan, Rao Yili, et al. The Clinical Value of Xpert MTB/RIF Test Todetect Mycobacterium Tuberculosis and Drug Resistance of Branchofiberoscope Lavage Fluid in Diagnosing of Tuberculosis [J]. Medical Recapitulate, 2016, 22(16): 3243-3246.

[11]. He Guiding, Li Tao, Shi Zheng, et al. Clinical performance of automated real-time nucleic acid amplification technology for rapid and simultaneous detection of tuberculosis and rifampicin resistance assay in diagnosis of pulmonary tuberculosis in 214 cases [J]. Chinese Journal of Infectious Diseases, 2016, 34(6):349-353. 